\title{
Tracheal tube cuff pressure monitoring: Assessing current practice in critically ill patients at Chris Hani Baragwanath Academic Hospital
}

\author{
A B Khan, ${ }^{1}$ MB BCh, FCPaed (SA), Cert Crit Care (SA), MMed; OrcID 0000-0002-3105-3522; \\ K Thandrayen, ${ }^{2} \mathrm{MB}$ BCh, FCPaed (SA), MMed, PhD, Cert Endocrinology and Metabolism (Paeds); OrcID 0000-0002-4028-2749; \\ S Omar, ${ }^{1}$ MB ChB, FCPath (SA), DA (SA), Cert Crit Care (SA); OrcID 0000-0001-8494-1518 \\ ${ }^{1}$ Department of Critical Care, Faculty of Health Sciences, University of the Witwatersrand, Johannesburg, South Africa \\ ${ }^{2}$ Department of Paediatrics, Faculty of Health Sciences, University of the Witwatersrand, Johannesburg, South Africa
}

Corresponding author: A B Khan (abatiya@gmail.com)

\begin{abstract}
Background. Intubated patients with a high tracheal tube cuff pressure (CP) are at risk of developing tracheal or subglottic stenosis. Recently an increasing number of patients have presented to our hospital with these complications.

Objectives. To determine the frequency of tracheal tube $\mathrm{CP}$ measurements and the range of $\mathrm{CP}$ and to explore nursing knowledge regarding $\mathrm{CP}$ monitoring.

Methods. Frequency of CP measurement was assessed using a prospective chart review, followed by an interventional component. In the final stage nurses completed a self-administered questionnaire.

Results. A total of 304 charts from 61 patients were reviewed. Patients' ages ranged from 1 to 71 years, with a male preponderance (1.5:1). The majority of charts (87\%) did not reflect a documented CP measurement and only 12 charts showed at least one measurement per shift. Only $17 \%$ of recorded CPs were within the recommended range; $59 \%$ were too low. The questionnaire was completed by only $51 \%$ of the 75 respondents. Nursing experience ranged from 3 to 35 years and $92 \%$ of respondents were trained in critical care. Knowledge of current critical care CP monitoring guidelines was reported by $62 \%$ of the respondents $(n=23 / 37)$. Only $53 \%(20 / 38)$ reported routinely measuring CP. Almost all respondents (94\%) knew of at least one complication of abnormal CP.

Conclusion. Having a basic knowledge of CP measurement, having awareness of the complications of abnormal CP and the availability of national best practice guidelines did not translate into appropriate ICU practice. Research into effective implementation strategies to achieve best practice is needed.

Keywords. Critically ill, cuff pressure monitoring, intensive care unit, paediatric cuff pressure monitoring, tracheal tube cuff pressures.
\end{abstract}

S Afr J Crit Care 2019;35(1):8-13. https://doi.org/10.7196/SAJCC.2019.v35i1.373

Contribution of study

- Basic knowledge of cuff pressure measurement may not always result in best practice.

- Improvement in current practice requires research into effective implementation strategies.

Cuffed tracheal tubes are used to prevent loss of tidal volume during positive pressure ventilation, minimise pulmonary aspiration of gastric and oral secretions, facilitate respiratory monitoring and, in the paediatric population, reduce the need for repeated laryngoscopy due to incorrect tube size..$^{[1-3]}$

These goals are achieved by appropriate cuff inflation. Cuff pressure (CP) should be $>25 \mathrm{cmH}_{2} \mathrm{O}$ to prevent aspiration and $<30 \mathrm{cmH}_{2} \mathrm{O}$ to avoid damage to surrounding structures. ${ }^{[4,5]}$ Obstruction to blood flow occurs when $\mathrm{CP}$ exceeds capillary perfusion pressure, resulting in ischaemia of the tracheal mucosa. Blood flow is impeded at CP $\geq 30 \mathrm{cmH}_{2} \mathrm{O}$ in normotensive adult patients, with total obstruction of flow occurring at $\mathrm{CP} \geq 50 \mathrm{cmH}_{2} \mathrm{O}^{[5]}$ No paediatric studies have been performed to assess capillary perfusion pressure or the $\mathrm{CP}$ at which tracheal capillary blood flow is impeded. The extent of damage from increased $\mathrm{CP}$ is related to the absolute pressure exerted by the cuff and the duration of this pressure (mucosal damage is noted to occur within 15 minutes of exposure to high pressures), with a greater contribution being from the absolute pressure. ${ }^{[5,6]}$ Injuries from high CP range from mucosal ulceration to tracheo-oesophageal fistula. ${ }^{[6,7]}$ Low CPs are also associated with risks, including the development of ventilator-associated pneumonia secondary to aspiration and compromised ventilation resulting from loss of positive pressure. ${ }^{[1,8]}$

Internationally accepted consensus guidelines for optimal CP range and frequency of measurement are lacking. ${ }^{[9]} \mathrm{A}$ local nursing guideline suggests a $\mathrm{CP}$ range of $25-30 \mathrm{cmH}_{2} \mathrm{O}^{\left[{ }^{[10]}\right.}$ The 2015 American Heart Association Pediatric Advanced Life Support guidelines recommend using the manufacturer's specification for appropriate $\mathrm{CP}$ in children $<9$ years and suggest a reference range of $20-25 \mathrm{cmH}_{2} \mathrm{O}^{[11]}$

$\mathrm{CP}$ should be measured using a manometer or pressure transducer, as techniques such as digital palpation and the minimal leak technique 
performed in isolation have been shown, in both adult and paediatric studies, to result in a $\mathrm{CP}$ outside the recommended range. ${ }^{[12-14]} \mathrm{A}$ survey on the minimal occlusion volume technique showed variation in technique, which may reduce efficacy. ${ }^{[15]}$ If pressures above $30 \mathrm{cmH}_{2} \mathrm{O}$ are required to achieve an adequate seal, the tracheal tube is likely to be too small and replacement is recommended. ${ }^{[16]}$

Ideally, pressures should be monitored continuously using pressure transducers and be adjusted accordingly. ${ }^{[17-19]}$ This method of CP measurement was validated by Sole et al. ${ }^{[20]}$ and has been shown to be superior to intermittent measurement to maintain $\mathrm{CP}$ within the recommended range in both adult and paediatric patients. ${ }^{[19-21]}$ This may allow for rapid adjustments to prevent time-dependent pressure complications such as mucosal injury ${ }^{[6]}$

Modern tracheal tubes, which are made from foam rubber or are fitted with pressure-relief valves or safety balloons, may provide alternatives to prevent complications. ${ }^{[6,22]}$ In the developing world, where limited resources may preclude the use of such tubes, the alternative is measuring CP intermittently (preferably every 6 hours). ${ }^{[17]}$ A South African (SA) nursing guideline suggests that $\mathrm{CP}$ should be measured once per shift (every 12 hours), which translates to twice daily. ${ }^{[10]}$ According to recommended standards, this would be an acceptable minimum to achieve an adequate seal by adjusting the pressure yet maintaining the pressure within the recommended safety limits. ${ }^{[10,23]}$

Numerous studies have shown that CP monitoring is poorly implemented and that pressures are consequently often higher than recommended ${ }^{[23-25]}$ or that measurements are not routinely performed. ${ }^{[2,27]}$ To avoid complications of overinflation, $\mathrm{CP}$ needs to be monitored using objective measures and documented regularly and routinely ${ }^{[10,23,28,29]}$ This requires staff awareness regarding care of cuffed tubes. Both local and international studies highlight the lack of awareness and misconceptions regarding endotracheal tube $\mathrm{CP}$ among staff in certain intensive care units (ICUs). ${ }^{[28,30]}$ Even in centres where awareness is high, there appears to be considerable heterogeneity regarding optimal target pressures and frequency of monitoring. ${ }^{[9,18]}$

Given the lack of standardisation in CP measurement, we investigated the current practices related to the monitoring of endotracheal or tracheostomy tube CP in our ICU.

\section{Methods \\ Study design}

A prospective cross-sectional chart review and interventional study of $\mathrm{CP}$ measurement and adjustment was undertaken at the main ICU of the Chris Hani Baragwanath Academic Hospital (CHBAH), SA. This multidisciplinary unit caters for both adult and paediatric patients. The study period spanned 50 consecutive days (12 June - 31 July 2017). All patients who had a cuffed tracheal tube in situ for $\geq 24$ hours were included; patients whose cuffs were deflated intentionally were excluded. The unit of measurement was patient days and therefore multiple readings were obtained from the same patient.

Ethical approval was obtained from the University of the Witwatersrand's Human Research Ethics Committee (ref. no. M160473). Informed consent and assent, as applicable, were obtained, initially from the next of kin and then also from the patient upon extubation and being competent to do so.

\section{Data collection}

The following information was collected from the chart review: demographic data; diagnostic categories; presence of an endotracheal or a tracheostomy tube; frequency of nurse-documented CP measurement.
All data were recorded on a collection form and subsequently captured electronically.

\section{Equipment and study procedure}

The interventional arm of the study involved CP measurement by the investigator using an aneroid manometer called a hand-pressure gauge (Covidien, Germany). The same make and model of manometer was used by the ICU staff. The unit of measurement was centimetres of water $\left(\mathrm{cmH}_{2} \mathrm{O}\right)$. The manometer was fitted with a pressure gauge, a bulb for inflation, a button for deflation and tubing to connect the manometer to the bulb of the tube's cuff. It was precalibrated and used according to the manufacturer's instructions, as also employed by the ICU staff.

The investigator checked $\mathrm{CP}$ once per 24-hour period, correcting the pressure if outside the recommended range.

Paediatric patients were divided into two age groups ( $<9$ years and $\geq 9$ years) owing to differences in recommended $\mathrm{CP}$ ranges. The range used for the purpose of the study was $25-30 \mathrm{cmH}_{2} \mathrm{O}$ for patients $\geq 9$ years and $20-25 \mathrm{cmH}_{2} \mathrm{O}$ for patients $<9$ years. ${ }^{[10,11]}$ All data were anonymised prior to analysis.

\section{Methods for nursing survey}

A voluntary, anonymous questionnaire was distributed among the full professional nursing complement present in the ICU over a 2-week period. The number of nursing staff available for participation during the study period was estimated at 96. An $80 \%$ response rate for completion of the questionnaires was predicted and the anticipated sample size was therefore 76 . The questionnaire was administered four times, once to each group of nurses (divided according to their shifts). Questionnaires were collected at the end of each shift to minimise bias. Consent was implied by return of a completed questionnaire. The nursing questionnaire collected information on demographics, education regarding $\mathrm{CP}$ monitoring, current practice and basic knowledge regarding $\mathrm{CP}$. Respondents were asked to place the completed questionnaire in a sealed box in the nurses' tearoom before the end of the shift.

The main outcome was to assess the frequency of $\mathrm{CP}$ measurements as documented by nurses and their knowledge of $\mathrm{CP}$ monitoring. To assess study-related bias, frequency of $\mathrm{CP}$ measurement in the first half of the study period was compared with that in the second half. We assessed the proportions of normal, low and high CPs as a secondary outcome.

\section{Statistical analysis}

A sample size of 400 patient days (across adult and paediatric patients) was calculated based on a 95\% confidence level, an expected proportion of $50 \%$ and a $5 \%$ precision level. ${ }^{[31]}$ Data analysis was performed using Statistica (version 13) (StatSoft, USA). Descriptive analysis of the categorical data involved frequency and percentage tabulation and was illustrated as bar charts. Continuous variables were assessed for normality and described using means and standard deviations (if deemed normally distributed) or medians and minimum/maximum value (for non-parametric data). A Mann-Whitney $U$ test was used to compare independent medians. A chi-squared test was used to compare percentages. A Spearman's correlation coefficient was used to assess correlation. A $p$-value $<0.05$ was considered to be statistically significant.

\section{Results}

\section{Chart review and cuff pressure measurements}

We screened 167 patients, of which 54 adult and 9 paediatric patients met the inclusion criteria. One adult and one paediatric patient were 
subsequently excluded as they refused consent. The final analysis therefore included 53 adult and 8 paediatric patients, from whom a total of 304 charts were reviewed (Fig. 1). Demographic data of the sample are provided in Table 1.

The chart review revealed 264 (87\%) charts reflecting no nursedocumented CP measurements (Fig. 2). Although 19 charts had more than one reading per day, further analysis showed that only 12 charts (4\%) reflected a nurse-documented CP measurement at least once per 12-hour shift, as suggested by guidelines.

Investigator-recorded CP measurements showed 83\% (252/304) of pressures to be outside of the recommended ranges and consequently required adjustment (Fig. 3).
As shown in Table 2, there was no significant difference between the percentage of nurse-documented $\mathrm{CP}$ measurements during the first half of the study (days $1-25)$ and the second half (days $26-50)(p=0.28)$. There was also no correlation (Spearman) between the frequency of nurse-documented CP measurements and study stage $(r=0.01)$.

No significant difference in frequency of nurse-documented $\mathrm{CP}$ measurements was found when data were analysed according to tube type $(6 \%$ and $8 \%$ for endotracheal and tracheostomy tubes, respectively; $p=0.72$ ). There was also no significant difference between the number of investigator-measured pressures found to be outside of the recommended range when analysed according to tube type (81\% and $90 \%$ for endotracheal and tracheostomy tubes, respectively; $p=0.12$ ).

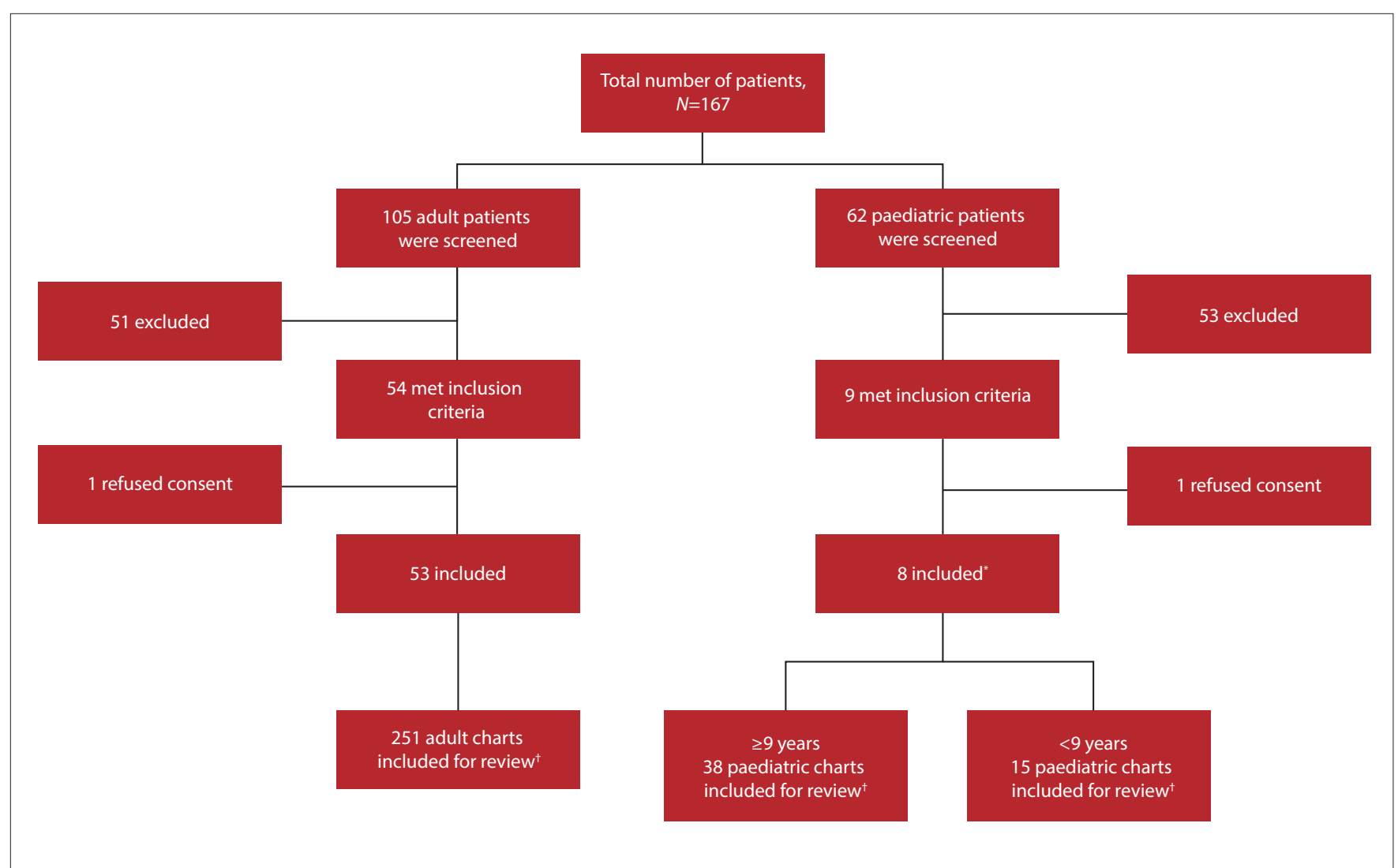

Fig. 1. Flow diagram illustrating sample selection.

${ }^{*}$ The paediatric group is divided according to age ( $<9$ years and $\geq 9$ years) owing to differences in the recommended cuff pressure ranges (the same range is used for adults and patients $\geq 9$ years).

'Number of charts obtained from included patients.

Table 1. Demographic data of the adult and paediatric patient groups

\begin{tabular}{|c|c|c|c|c|}
\hline & \multirow[b]{2}{*}{ All } & \multirow{2}{*}{$\begin{array}{l}\text { Adult patients } \\
\text { ( } \geq 18 \text { years) }\end{array}$} & \multicolumn{2}{|c|}{ Paediatric patients } \\
\hline & & & $<9$ years $^{\star}$ & $9-17$ years \\
\hline Patients, $n(\%)$ & $61(100)$ & $53(87)$ & $4(7)$ & $4(7)$ \\
\hline Charts, $n$ & 304 & 251 & 15 & 38 \\
\hline Age (years), mean (SD) & $39(17.4)$ & $43(14)$ & $4(2.6)$ & $16(1.3)$ \\
\hline Weight $(\mathrm{kg})$, mean $(\mathrm{SD})$ & $72(21)$ & $77(15.5)$ & $18(7.1)$ & $55(4.7)$ \\
\hline Sex, male (\%) & 61 & 53 & 5 & 3 \\
\hline \multicolumn{5}{|l|}{ Diagnostic category, $n$} \\
\hline Trauma & 26 & 24 & 2 & 0 \\
\hline Medical & 20 & 16 & 1 & 3 \\
\hline Surgical & 13 & 11 & 1 & 1 \\
\hline Other $^{\dagger}$ & 2 & 2 & 0 & 0 \\
\hline
\end{tabular}




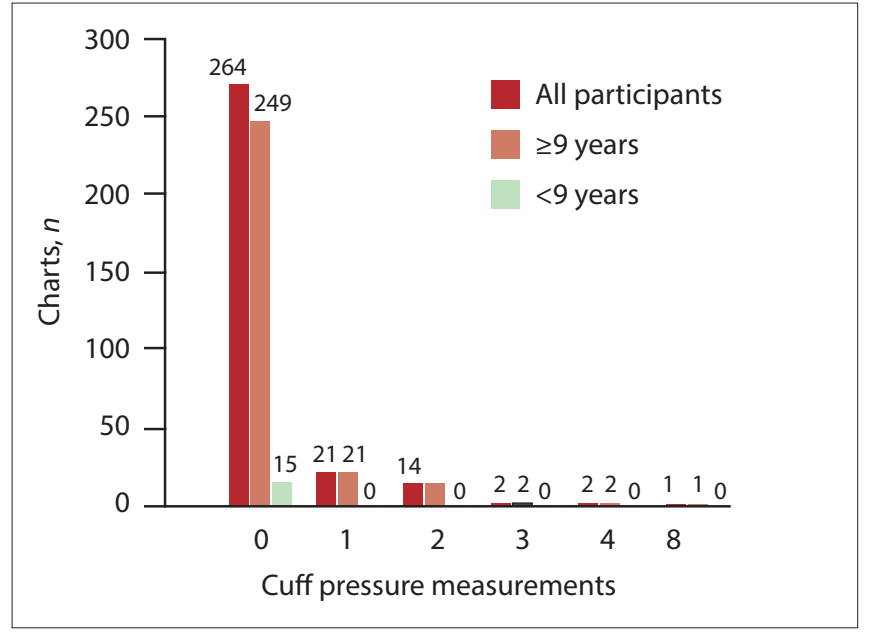

Fig. 2. Frequency of cuff pressure measurements documented by nurses on patient chart per day. As the same pressure range is recommended for adults and patients $\geq 9$ years, results are divided accordingly.

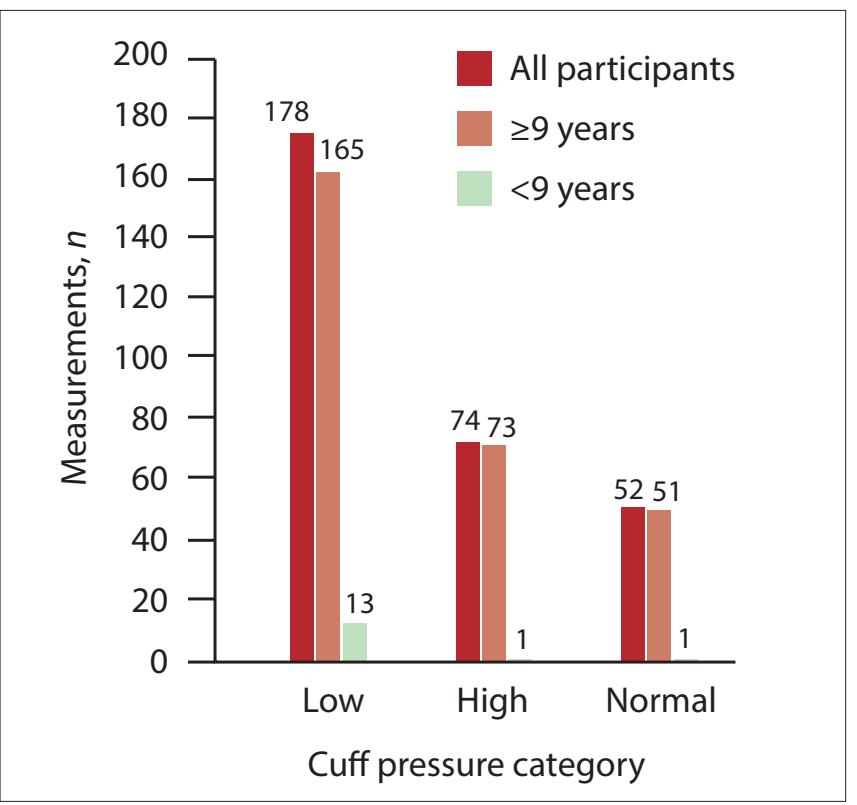

Fig. 3. Results of cuff pressure control checks performed by investigator. As the same pressure range is recommended for adults and patients $\geq 9$ years, results are divided accordingly.

Table 2. Cuff pressure measurements documented by nurses over the 50-day study period

\begin{tabular}{llll}
\hline Stage of study & Yes, $\boldsymbol{n}(\%)$ & No, $\boldsymbol{n}(\%)$ & $\boldsymbol{p}$-value \\
\hline Stage 1 (day 1 - day 25) & $25(15)$ & $141(85)$ & 0.28 \\
Stage 2 (day 26 - day 50) & $15(11)$ & $123(89)$ & \\
${ }^{*}$ As calculated from $\chi^{2}$-test. & & &
\end{tabular}

\section{Nursing questionnaire}

The overall response rate was 51\% (38/75). As questions were analysed individually, the total counts used in the subanalyses of individual questions may be less than 38 in some cases. All respondents were professional nurses and 92\% (35/38) were certified for critical care. Only one respondent was not a permanent staff member. The mean (and associated standard deviation) for ICU experience was 14.8 (8.6) years, based on a total of 30 responses (eight respondents did not state the extent of their ICU experience). The majority of these respondents $(87 \% ; 26 / 30)$ had $\geq 5$ years' ICU experience.
Approximately three-quarters $(76 \% ; 29 / 38)$ of the respondents had training in $\mathrm{CP}$ monitoring. Knowledge of existing CP monitoring guidelines was reported in $62 \%$ of the responses (23/37). Only $53 \%$ respondents (20/38) reported routinely measuring CPs. Approximately a third of the respondents $(34 \% ; 13 / 38)$ did not respond to the question regarding the frequency of their CP measurements, whereas $48 \%$ (18/38) reported measuring $\mathrm{CP}$ at least twice a day, $8 \%$ (3/38) reported measuring once a day and $10 \%(4 / 38)$ reported measuring at their own discretion.

In response to the question regarding the method used for $\mathrm{CP}$ measurement, 93\% of respondents (25/27) indicated the use of a manometer; 11 respondents did not answer the question. The $\mathrm{CP}$ range $\left(25-30 \mathrm{cmH}_{2} \mathrm{O}\right.$ ) stated by the local nursing guideline was correctly reported by $68 \%$ of the respondents (26/38), although only 7 respondents knew the correct unit of measurement. Almost all of the respondents (95\%; 35/37) were aware of complications of a low CP. Displacement or dislodgement of the tube was reported as a complication by $60 \%$ of respondents (22/37). Two respondents did not answer the question regarding complications of high CP. Those who did respond were all $(36 / 36)$ aware of the complications of high CP.

\section{Discussion}

It is important to maintain $\mathrm{CP}$ within the recommended range to prevent patient morbidity and mortality. ${ }^{[1,6,8,29,32]}$ Our data indicate very few CP measurements within the recommended range. This places the majority of our patients (in both the adult and paediatric groups) at risk for preventable complications, regardless of type of tube. Similar low rates of $\mathrm{CP}$ measurements were found in a survey conducted in Cape Town, SA, and also in an international study, which may be a reflection of the lack of international consensus guidelines for $\mathrm{CP}$ measurement. ${ }^{[27,33]}$

The first arm of the study involved a chart review to assess the frequency of nurses' $\mathrm{CP}$ measurements over a 24-hour period. The review showed that $\mathrm{CP}$ had not been documented in the majority of cases $(87 \%)$. Of the 40 charts on which pressures were indicated, only 19 showed measurements recorded twice or more during a 24-hour period, as required by local guidelines. However, as the national nursing guideline recommends one reading per shift, we further analysed these charts and found that in patients with multiple readings, only $12(4 \%)$ had readings recorded at least once per shift. The remaining 7 charts showed multiple readings in one 12-hour period but none in the other. A Polish study found that $\mathrm{CP}$ was not measured routinely in their controlled units. ${ }^{[27]}$ Similarly, a study conducted in Cape Town, SA, showed CP measurements performed twice daily in only $15 \%$ of patients, with $\mathrm{CP}$ monitors being used in only $38 \%$ of their patients. ${ }^{[33]}$ Of note in our study is that nurses documented the CP they set rather than what they measured, so the number of abnormal readings found by nurses could not be determined.

The interventional arm of the study involved investigator-recorded $\mathrm{CP}$ checks for every chart review per patient to determine whether the $\mathrm{CP}$ was within an acceptable range. ${ }^{[10,11]}$ Only $17 \%$ of the recorded pressures were within the accepted range; the majority (59\%) were too low. However, when the reference range cited in international studies $\left(20-30 \mathrm{cmH}_{2} \mathrm{O}\right)$ was used, ${ }^{[9,34]}$ the number of investigatorchecked pressure readings within an acceptable range improved to $36 \%$. Unfortunately an acceptable range for $\mathrm{CP}$ has yet to be validated and hence heterogeneity exists in the reported values. Some studies stated only an upper limit of pressure, commonly $30 \mathrm{cmH}_{2} \mathrm{O} .^{[28,30]}$ Our findings highlight the importance of regular CP measurements, as $83 \%$ of readings being outside the target range means that $83 \%$ of patients were at risk for complications from abnormal CP. The finding of 
predominantly low pressures was not unexpected, as CP decreases over time and can be noted from as early as 4 hours post inflation. ${ }^{[35]}$

As CP measurement is performed largely by the nursing staff, it is imperative that they have a working knowledge of $\mathrm{CP}$ monitoring. With this in mind, a nursing questionnaire was administered to the nursing staff of our ICU. The response rate was only $51 \%$, which may be a reflection of the poor $\mathrm{CP}$ measurement practice observed in the first part of the study. In view of the poor response rate, findings are not representative of the ICU staff at large. Despite this limitation, the findings provide some insights and are therefore described. A third of respondents were not aware of guidelines related to CP monitoring and almost half reported that they did not document $\mathrm{CP}$ routinely. Two-thirds of respondents reported the misconception that the cuff of an endotracheal tube serves to keep the tube in place and cited tube dislodgement as a complication of low CP. Mol et al. ${ }^{[30]}$ reported a similar misconception from their study. The overall awareness of complications due to abnormal CP was high (>90\%). A survey performed at a Flemish conference in 2014 revealed a similar response profile to what we observed in our study: although $80 \%$ of respondents had ICU qualifications and $50 \%$ had $>10$ years' experience, $66 \%$ did not know the $\mathrm{CP}$ range despite a large number of respondents being aware of the complications of abnormal CP. ${ }^{[34]}$ This implies a knowledge gap and, in addition, a gap between knowledge and application in respondents who do have some knowledge. This has been well described in the nursing fraternity. ${ }^{[36,37]}$ We postulate a lack of unit-specific, evidence-based guidelines on tracheal tube care, a lack of resources (insufficient number of manometers) and poor access to necessary equipment (manometers not at the bedside) as possible reasons for the current practice. A survey performed in ICUs in Australia and New Zealand showed that of the 92 ICUs surveyed, only 34 (37\%) had formalised written protocols on cuff management, resulting in variable practice. ${ }^{[38]}$ Only $76 \%$ of respondents in our study reported having been trained on CP measurement, which may represent another barrier to consistent practice. Although formulating and implementing an evidence-based guideline for our ICU may raise awareness regarding $\mathrm{CP}$ measurement, studies have shown that without ongoing educational programmes to improve knowledge, implementing protocols or guidelines is insufficient for reducing complications. ${ }^{[31]}$ Findings of a Malawian study that reviewed the practical effect of implementing a CP management guideline showed that nurses acknowledged the existence of the guideline post implementation but some seldom used it and a notable percentage of nurses continued to use varying and non-recommended practices of $\mathrm{CP}$ measurement. ${ }^{[39]}$

\section{Study limitations}

Limitations of this study included a poor response rate in the nurses' survey, which means that the information gained was not representative of the nursing workforce. Piloting or validating the questionnaire prior to use may have added to the value of the results obtained. Although the study period was extended to 50 days (from the initially planned 30 days), the calculated sample size for a precision level of 5\% was not reached owing to lower-than-predicted bed occupancy and intubation rates during the study period. Given the trend of the results over time, it appears unlikely that an additional 96 measurements would make a meaningful change to the outcomes. Although this reduced the precision of the study, the results still yield useful insights into current practice in our unit and provide a basis for instituting change. Although concern was raised about the investigator's performing CP measurements resulting in a change in practice and so introducing bias as the study progressed, we did not find an association between the frequency of nurse-documented CP measurements and study stage. Ours is the first study in SA to combine analysis of CP monitoring practice with a nursing survey. We have shown that despite nursing staff's knowledge of the potential harm of abnormal CP, care is still suboptimal, which may be reflective of ineffective implementation processes.

\section{Conclusion}

Despite basic knowledge of $\mathrm{CP}$ measurement, awareness of the complications of abnormal $\mathrm{CP}$ and national best practice guidelines being available, these measures did not translate into appropriate ICU practice. Research into effective implementation strategies to achieve best practice is much needed.

Acknowledgements. Prof. RL Mathivha (CHBAH and University of the Witwatersrand) is thanked for her advice and support during the study and in completing the manuscript. We also thank Ms Petra Gaylard (Data Management and Statistical Analysis) for her assistance with statistical planning during protocol development.

Author contributions. ABK was the principal investigator and responsible for all aspects of the research. KT and SO were the study supervisors and contributed to data analysis and interpretation and critical revision of both the protocol and the final manuscript.

Funding. Manometers were supplied on a loan basis by the local suppliers. Conflicts of interest. None.

1. Main E, Castle R, Stocks J, James I, Hatch D. The influence of tracheal tube leak on the assessment of respiratory function in ventilated children. Intensive Care Med 2001;27(11):1788-1797. https:// doi.org/10.1007/s001340101105

2. Khine HH, Corddry DH, Kettrick RG, et al. Comparison of cuffed and uncuffed endotracheal tubes in young children during general anesthesia. Anesthesiology 1997;86(3):627-631. https:// doi.org/10.1097/00000542-199703000-00015

3. Weiss M, Dullenkopf J, Fischer A, Keller C, Gerber AC, European Paediatric Endotracheal Intubation Study Group. Prospective randomized controlled multi-centre trial of cuffed or uncuffed endotracheal tubes in small children. Br J Anaesth 2009;103(6):867-873. https://doi. org/10.1093/bja/aep290

4. Bernhard WN, Cottrell JE, Sivakumaran C, Patel K, Yost L, Turndorf H. Adjustment of intracuff pressure to prevent aspiration. Anesthesiology 1979;50(4):363-365. https://doi. org/10.1097/00000542-197904000-00018

5. Seegobin RD, Van Hasselt GL. Endotracheal cuff pressure and tracheal mucosal blood flow: Endoscopic study of effects of four large volume cuffs. Br Med J (Clin Res Ed) 1984;288(6422):965968. https://doi.org/10.1136/bmj.288.6422.965

6. Leigh JM, Maynard JP. Pressure on the tracheal mucosa from cuffed tubes. Br Med J 1979;1(6172):1173-1174. https://doi.org/10.1136/bmj.1.6172.1173

7. Sultan P, Carvalho B, Rose BO, Cregg R. Endotracheal tube cuff pressure monitoring: A review of the evidence. J Perioper Pract 2011;21(11):379-386. https://doi.org/10.1177/175045891102101103

8. Nseir S, Lorente L, Ferrer M, et al. Continuous control of tracheal cuff pressure for VAP prevention: A collaborative meta-analysis of individual participant data. Ann Intensive Care 2015;5:43. https://doi.org/10.1186/s13613-015-0087-3

9. Talekar CR, Udy AA, Boots RJ, Lipman J, Cook D. Tracheal cuff pressure monitoring in the ICU: A literature review and survey of current practice in Queensland. Anaesth Intensive Care 2014;42(6):761-770. https://doi.org/10.1177/0310057x1404200612

10. Perrie H. Nesibopho Best Practice Guideline on Tracheal Tube Cuff Pressure Monitoring Johannesburg: Nesibopho Healthcare, 2010.

11. De Caen AR, Berg MD, Chameides L, et al. Part 12: Pediatric advanced life support: 2015 American Heart Association guidelines update for cardiopulmonary resuscitation and emergency
cardiovascular care. Circulation 2015;132(18 Suppl 2):S526-S542. https://doi.org/10.1161/ cardiovascular care. Cir

12. Zand F, Nekooeian AA, Rohani M. Endotracheal tube cuff pressure monitoring in intensive care units. Iran Red Crescent Med J 2008;10(3):223-227.

13. Harvie DA, Darvall JN, Dodd M, et al. The minimal leak test technique for endotracheal cuff maintenance. Anaesth Intensive Care 2016;44(5):599-604. https://doi. org/10.1177/0310057x1604400512

14. Tobias JD. Pediatric airway anatomy may not be what we thought: Implications for clinical practice and the use of cuffed endotracheal tubes. Pediatr Anesth 2015;25(1):9-19. https://doi. org/10.1111/pan.12528

15. Rose L, Redl L. Minimal occlusive volume cuff inflation: A survey of current practice. Intensive Crit Care Nurs 2008;24(6):359-365. https://doi.org/10.1016/j.iccn.2008.05.002

16. Salgo B, Schmitz A, Henze G, et al. Evaluation of a new recommendation for improved cuffed tracheal tube size selection in infants and small children. Acta Anaesthesiol Scand 2006;50(5):557561. https://doi.org/10.1111/j.1399-6576.2006.01003.x

17. Memela ME, Gopalan PD. Variations in endotracheal tube cuff pressure: Is 8-hourly monitoring enough? S Afr J Crit Care 2014;30(2):35-40. https://doi.org/10.7196/SAJCC.159

18. Gopalakrishnan S, Barry N, Rice J, Tobias JD. Cuffed endotracheal tubes in infants and children: A technique to continuously measure the intracuff pressure. Int J Pediatr Otorhinolaryngol 2013;77(7):1135-1138. https://doi.org/10.1016/j.ijporl.2013.04.020 
19. Sole ML, Su X, Talbert S, et al. Evaluation of an intervention to maintain tube cuff pressure within therapeutic range. Am J Crit Care 2011;20(2):109-117. https://doi.org/10.4037/ajcc2011661

20. Sole ML, Aragon D, Bennett M, Johnson RL. Continuous measurement of endotracheal tube cuff pressure: How difficult can it be? AACN Adv Crit Care 2008;19(2):235-243. https://doi. org/10.1097/01.AACN.0000318126.79630.76

21. Vottier G, Matrot B, Jones P, Dauger S. A cross-over study of continuous tracheal cuff pressure monitoring in critically-ill children. Intensive Care Med 2016;42(1):132-133. https://doi. org/10.1007/s00134-015-4103-8

22. Power KJ. Foam cuffed tracheal tubes: Clinical and laboratory assessment. Br J Anaesth 1990;65(3):433-437. https://doi.org/10.1093/bja/65.3.433

23. Braz JR, Navarro LH, Takata IH, Nascimento Júnior P. Endotracheal tube cuff pressure: Need for precise measurement. Sao Paulo Med J 1999;117(6):243-247. https://doi.org/10.1590/s151631801999000600004

24. Morris LG, Zoumalan RA, Roccaforte JD, Amin MR. Monitoring tracheal tube cuff pressures in the intensive care unit: A comparison of digital palpation and manometry. Ann Otol Rhinol Laryngol 2007;116(9):639-642. https://doi.org/10.1177/000348940711600902

25. Tobias JD, Schwartz L, Rice J, Jatana K, Kang DR. Cuffed endotracheal tubes in infants and children: Should we routinely measure the cuff pressure? Int J Pediatr Otorhinolaryngol 2012;76(1):61-63. https://doi.org/10.1016/j.ijporl.2011.09.033

26. Jordan P, Van Rooyen D, Venter D. Endotracheal tube cuff pressure management in adult critical care units. S Afr J Crit Care 2012;28(1):13-16. https://doi.org/10.7196/SAJCC.129

27. Wujtewicz MA, Sawicka W, Sein Anand J, et al. [Monitoring of tracheal tube cuff pressure in patients treated in intensive therapy unit and intensive care units]. Przegl Lek 2004;61(4):353-355.

28. Bhatta K, Greer R. Awareness and monitoring of tracheal tube cuff pressure in a multidisciplinary intensive care unit. Anaesth Intensive Care 2007;35(2):302-303.

29. Hockey CA, Van Zundert AAJ, Paratz JD. Does objective measurement of tracheal tube cuff pressures minimise adverse effects and maintain accurate cuff pressures? A systematic review and meta-asolys. Anesth Intensive Care 2016:44(5):560-570. review and meta-analysis. Anaesth Intensive Care 2016;44(5):560-570. https://doi.
org/10.1177/0310057X1604400503
30. Mol DA, De Villiers G du T, Claassen AJ, Joubert G. Use and care of an endotracheal/ tracheostomy tube cuff - are intensive care unit staff adequately informed? S Afr J Sur 2004;42(1):14-16

31. Daniel WW. Biostatistics: A Foundation for Analysis in the Health Sciences. 10th edition. New York: John Wiley \& Sons, 2013.

32. Taylor C, Subaiya L, Corsino D. Pediatric cuffed endotracheal tubes: An evolution of care. Ochsner J 2011;11(1):52-56.

33. Raynham OW, Lubbe DE, Fagan JJ. Tracheal stenosis: Preventable morbidity on the increase in our intensive care units. S Afr Med J 2009;99(9):645-646.

34. Labeau SO, Bleiman M, Rello J, Vandijk DM, Claes B, Blot SI. Knowledge and managemen of endotracheal tube cuffs. Int J Nurs Stud 2015;52(1):498-499. https://doi.org/10.1016/j. ijnurstu.2014.09.010

35. Sole ML, Combs SM, Willis J. Changes in endotracheal cuff pressures over time. Crit Care Med 2003;31(Suppl 2):A144.

36. Hussein MTEL, Osuji J. Bridging the theory-practice dichotomy in nursing: The role of nurse educators. J Nurs Educ Pract 2017;7(3):20-25. https://doi.org/10.5430/jnep.v7n3p20

37. Perrie H, Schmollgruber S, Bruce JC, Becker PJ. Knowledge of intensive care nurses in selected care areas commonly guided by protocols. S Afr I Crit Care 2014:30(1):14-18. https://doi. org/10.7196/SAJCC. 158

38. Rose L, Redl L. Survey of cuff management practices in intensive care units in Australia and New Zealand. Am J Crit Care 2008;17(5):428-435.

39. Mpasa F. Management of endotracheal tube cuff pressure in mechanically ventilated adult patients in intensive care units in Malawi. PhD thesis. Port Elizabeth: Nelson Mandela Metropolitan University, 2017:1-239. http://hdl.handle.net/10948/19673

Reviewed 13 May 2019. Accepted 22 May 2019. 